\title{
Observation of the Photon-Blockade Breakdown Phase Transition
}

\author{
J. M. Fink, ${ }^{1,2, *}$ A. Dombi, ${ }^{3}$ A. Vukics, ${ }^{3}$ A. Wallraff, ${ }^{1}$ and P. Domokos ${ }^{3, \dagger}$ \\ ${ }^{1}$ Department of Physics, ETH Zürich, CH-8093 Zürich, Switzerland \\ ${ }^{2}$ Institute of Science and Technology Austria, 3400 Klosterneuburg, Austria \\ ${ }^{3}$ Wigner Research Centre for Physics, H-1525 Budapest, P.O. Box 49, Hungary \\ (Received 13 August 2016; revised manuscript received 13 December 2016; published 31 January 2017)

\begin{abstract}
Nonequilibrium phase transitions exist in damped-driven open quantum systems when the continuous tuning of an external parameter leads to a transition between two robust steady states. In second-order transitions this change is abrupt at a critical point, whereas in first-order transitions the two phases can coexist in a critical hysteresis domain. Here, we report the observation of a first-order dissipative quantum phase transition in a driven circuit quantum electrodynamics system. It takes place when the photon blockade of the driven cavity-atom system is broken by increasing the drive power. The observed experimental signature is a bimodal phase space distribution with varying weights controlled by the drive strength. Our measurements show an improved stabilization of the classical attractors up to the millisecond range when the size of the quantum system is increased from one to three artificial atoms. The formation of such robust pointer states could be used for new quantum measurement schemes or to investigate multiphoton phases of finite-size, nonlinear, open quantum systems.
\end{abstract}

DOI: 10.1103/PhysRevX.7.011012

The prototype of a nonlinear quantum system is the one described by the Jaynes-Cummings (JC) model of a twolevel system coupled to a harmonic oscillator [1]. This model corresponds to very high accuracy to cavity quantum electrodynamics (QED) systems [2], where atomic dipole transitions are coupled to quasiresonant radiation modes of a resonator, or to circuit QED systems [3], where artificial atoms made of superconducting Josephson junctions are coupled to on-chip microwave resonators. The confinement of the photon into a small resonator volume results in a very strong coupling to the atom. Although the radiation mode spectrum is the well-known harmonic ladder, the coupling changes this to the significantly anharmonic JC spectrum, which allows for designing nonlinear processes within the low-intensity quantum domain and by means of only a single atom as a nonlinear medium in the resonator.

For monochromatic external driving with moderate power, the well-resolved resonances within the anharmonic JC spectrum [4-7] realize effectively a set of independent two-level systems, each of which can be selectively addressed. When tuning the cavity driving to resonance

\footnotetext{
* Corresponding author. jfink@ist.ac.at

Corresponding author. domokos.peter@wigner.mta.hu

Published by the American Physical Society under the terms of the Creative Commons Attribution 4.0 International license. Further distribution of this work must maintain attribution to the author(s) and the published article's title, journal citation, and DOI.
}

Subject Areas: Condensed Matter Physics, Quantum Physics, Superconductivity

with the lowest-lying excited state, at most a single photon can be in the resonator. This effect was named photon blockade [8] in analogy with Coulomb blockade for electrons in a quantum well, and was experimentally demonstrated by observing photon antibunching in the transmitted radiation [9-11]. Photon blockade is, in fact, more than the single-photon effect. The next manifold containing two energy quanta can be used as a two-photon gateway [12], and the concept can be further generalized to higher-order multiphoton transitions.

The discrete quantum system with its anharmonic spectrum cannot be excited at any frequency away from the well-resolved resonances. It is then fully reflective and stays in a dim state close to the ground state. When increasing the power of the drive, the effective two-level system undergoes power broadening by saturation and the disjoint set of off-resonant frequency ranges shrinks until the broadened resonances merge into a single broad structure [13]. However, even for very large drive powers, a photon-blockade frequency domain persists near the mode (here, we assume, for simplicity, resonance between the cavity mode and the atomic transition). In this frequency range, interestingly, the further enhancement of the drive power breaks the photon blockade in a first-order phase transition [14].

In first-order phase transitions, the two stable phases can coexist in a critical range of parameter values. Quite typically, it is the direction from which the control parameter is tuned into the critical domain that determines which phase the system is prepared in. At a quantum mechanical level, this well-known hysteresis effect gives rise to continuously 
varying weights in a bimodal phase space distribution of the quantum system, as one of the classical attractors takes over by tuning the control parameter. This kind of transition takes place between the reflective vacuum state and a highly excited semiclassical state in the breakdown of the photonblockade regime $[13,14]$.

The coexistence of the two robust states with different macroscopic attributes is qualitatively different from a second-order phase transition, which can also exist in nonequilibrium settings, i.e., in a damped-driven open quantum system. In second-order transitions the two phases are sharply separated by a critical point where the quantum fluctuations must diverge so that the two phases with different symmetries can be matched. The self-organization of a Bose-Einstein condensate in an optical cavity [15] belongs to this class and has been experimentally observed [16].

A first-order dissipative quantum phase transition has been predicted in a wide variety of systems such as the vortex formation of a stirred Bose-Einstein condensate [17] or the deflection-induced coupling between an electron spin and a mechanical resonator [18]. Very recently, spatial symmetry breaking and hysteresis have been observed in the equilibrium state of an ultracold gas trapped in a double-well potential [19]. Dynamic optical hysteresis of a semiconductor microcavity for various sweep rates of the driving power has been demonstrated [20]. Such a dissipative phase transition is accompanied by long switching times, which also just now have been observed in a one-dimensional circuit QED lattice [21]. The associated bimodal phase space distribution, which we explore in zero dimensions, has not yet been observed, to the best of our knowledge.

In the case of the driven JC model, highly nonlinear dynamics and multistability can occur in various parameter regimes that lead to distinct phenomenologies. In microscopic quantum systems, such as a single-atom cavity QED system, one can refer to phases because one of the subsystems is a harmonic oscillator with infinitely many states $[22,23]$. Therefore, the highly excited part of the JC spectrum is quasiharmonic and can host coherent states or weakly phase-stretched coherent states due to the residual anharmonicity. These states can be robust stationary states, i.e., semiclassical attractors of the damped-driven system, where the environment brings in infinitely many degrees of freedom. The specific feature of the photon-blockade breakdown phase transition is that the semiclassical phases are separated by an inherently quantum effect. The photon blockade relies on the discrete, anharmonic spectrum in an extended range of the excitations, which has no classical counterpart. This is in contrast to the well-known optical bistability, which has a well-defined semiclassical limit [24]. The absorptive bistability in cavity QED realizations of the JC model with few atoms in the strong-coupling regime $[25,26]$ still preserves the semiclassical mean-field features that can be interpreted in terms of a classical saturable absorber. Moreover, in an atomic cavity QED system the coupling strength does not allow for resolved semiclassical attractors in the single-atom limit [27,28].

In the context of circuit QED, the dispersive limit of the JC bistability has been proposed for high-fidelity qubit readout [29], and it was shown to improve single-qubit measurements [30-32], similar to latching readouts with nonlinear cavities based on additional Josephson circuit elements $[33,34]$. Even if the qubit coupling to the mode is much larger than in atomic cavity QED systems, the large detuning softens the nonlinearity. Hence, quantization of the resonance frequency shift has not been resolved at the high photon numbers required for this readout $\left(\sim 10^{5}\right)$ and the bistability behavior can be interpreted within a semiclassical Duffing-type oscillator model with a single intensity-dependent refractive index. As a consequence, the observed transition from low to high input power is continuous, a single-valued function fully compatible with a semiclassical description of the effect. Note also that the switching of the transmitted intensity signal in the singleatom measurement scheme [31,32] at constant input power is due to qubit state changes (quantum jumps). In contrast, in the photon-blockade phase transition the semiclassical attractors are left by a slow quantum diffusion process and the system is then driven into the other attractor's basin by the nonlinear JC dynamics.

The photon-blockade phase transition in the JC model has an intricate relation to the so-called spontaneous dressed state polarization [35]. The latter takes place in the resonantly driven resonant JC system with a nondecaying twolevel atom via a second-order transition above a threshold drive strength. Its remnants in the form of phase flipping have been observed in the homodyne measurement of light transmitted through a Fabry-Perot cavity, interacting with laser cooled ${ }^{133} \mathrm{Cs}$ atoms [36]. It was argued in Ref. [14] that a first-order transition via bistability is expected away from resonant driving. However, in the case of a nondecaying atom, $\gamma=0$, the semiclassical limit of the qubit variable is confined to the surface of the Bloch sphere. By contrast, for $\gamma \neq 0$, the highly excited phase of the optical bistability as well as that of the photon-blockade breakdown phase transition involves a completely saturated two-level system; i.e., the semiclassical spin variable contracts into the origin of the Bloch sphere.

A nonstationary, dynamical phase transition has been observed in a coupled JC dimer [37], but the detailed study of the stationary steady-state transition is hindered both experimentally and numerically by the very large critical photon numbers in a resonantly driven JC model. This obstacle can be avoided with a relatively simple trick. We show that coupling a third atomic level into the dynamics drastically changes the excitation route to the highly excited attractor and the transition regime between the two phases can be explored in detail.

A suitable experimental setting to realize the three-level system and the necessary strong-coupling strength is 
superconducting circuit QED, which offers large dipole coupling strengths and long coherence times of multiple superconducting artificial atoms embedded in a high-quality on-chip microwave cavity [38]. Our experimental device and setup is similar to the one in Ref. [39] and consists of three frequency tunable superconducting artificial atoms positioned at the antinodes of the first harmonic voltage standing wave resonance of a coplanar superconducting resonator at frequency $\omega_{c} /(2 \pi)=7.024 \mathrm{GHz}$; see Fig. 1(a).

We use capacitively shunted superconducting charge qubits, or transmon qubits [40], which have a flat charge dispersion but also a limited absolute anharmonicity of $\omega_{f e}-\omega_{e g} \simeq-E_{C} / \hbar$. Here, $|g\rangle,|e\rangle$, and $|f\rangle$ are the first three levels of the artificial atom, see Fig. 1(b), which all take part in the dynamics of the presented experiments. $E_{C} / h=$ $(459,359,358) \mathrm{MHz}$ are the charging energies and $g_{1} /(2 \pi)=(-52.7,55.4,55.8) \mathrm{MHz}$ the single-photon $|g\rangle$ to $|e\rangle$ transition to resonator coupling strengths of the atoms 1,2 , and 3. Similar to a harmonic oscillator, the coupling strength of the second excited level can be approximated [40] as $g_{2} \simeq \sqrt{2} g_{1}$. Using externally applied and locally concentrated magnetic fields, we can control the flux $\Phi$ through the individual transmon SQUID loops and change their Josephson energy $E_{J}(\Phi)$. This allows us to independently control the transmons' transition energies $\hbar \omega_{\text {eg }} \simeq$ $\sqrt{8 E_{C} E_{J}(\Phi)}-E_{C}$ and, in particular, to tune them in and out of resonance with the microwave resonator with a full width at half maximum linewidth of $\kappa /(2 \pi)=0.47 \mathrm{MHz}$. For most of this paper, we study the situation where two of the three artificial atoms are far detuned $\left(\omega_{e g} \ll \omega_{r}\right)$ and not interacting with the microwave resonator.

The Hamiltonian of the system in a frame rotating at the angular frequency $\omega$ of an external drive reads

$$
\begin{aligned}
H / \hbar= & \Delta_{1}|g\rangle\left\langle g\left|-\Delta_{2}\right| f\right\rangle\langle f|-\Delta_{c} a^{\dagger} a+\eta\left(a+a^{\dagger}\right) \\
& +g_{1}\left(a^{\dagger}|g\rangle\langle e|+| e\rangle\langle g| a\right) \\
& +g_{2}\left(a^{\dagger}|e\rangle\langle f|+| f\rangle\langle e| a\right),
\end{aligned}
$$
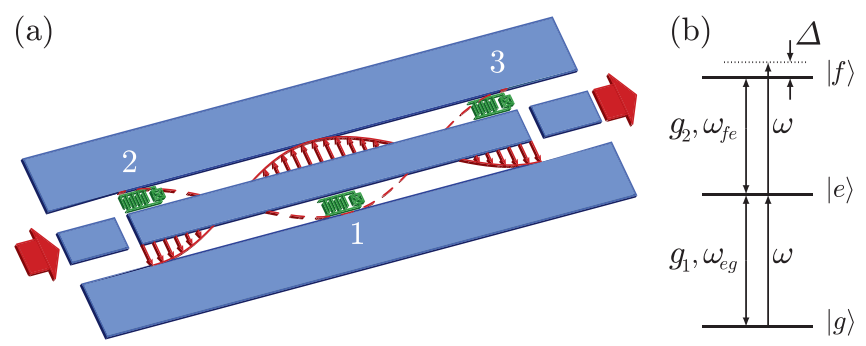

FIG. 1. (a) Scheme of the driven coplanar waveguide resonator (blue) coupled to up to three transmon artificial atoms (green). An external drive (red) is applied via the input capacitor, and the coherent transmission is detected via the output capacitor. (b) Level scheme of one artificial atom indicating the parameters of the Hamiltonian in Eq. (1). where the detunings are defined as $\Delta_{1}=\omega-\omega_{e g}$, $\Delta_{2}=\omega-\omega_{f e}$, and $\Delta_{c}=\omega-\omega_{c}$, and $\eta$ is the effective amplitude of the drive field. For the drive frequency, we consider the special case $\Delta_{1}=0$, as shown schematically in Fig. 1(b). We assume resonance also between the resonator mode and the $|g\rangle \leftrightarrow|e\rangle$ transition; i.e., $\Delta_{c}=0$. The remaining detuning is denoted by $\Delta_{2} \equiv \Delta$, which is then simply the charging energy $E_{C} / \hbar$. The dissipative processes are accounted for by the following Lindblad terms in the master equation of the density matrix:

$$
\begin{aligned}
\dot{\rho}= & -\frac{i}{\hbar}[H, \rho]+\frac{\kappa}{2}\left(2 a \rho a^{\dagger}-a^{\dagger} a \rho-\rho a^{\dagger} a\right) \\
& +\gamma_{\|}(2|g\rangle\langle e|\rho| e\rangle\langle g|-| e\rangle\langle e|\rho-\rho| e\rangle\langle e|) \\
& +2 \gamma_{\|}(2|e\rangle\langle f|\rho| f\rangle\langle e|-| f\rangle\langle f|\rho-\rho| f\rangle\langle f|) \\
& +8 \gamma_{\perp}\left(\left[\sum_{i=g, e, f}|i\rangle\langle i|\rho| i\rangle\langle i|\right]-\rho\right) .
\end{aligned}
$$

The rate $\kappa$ characterizes the cavity-photon loss due mainly to the photon outcoupling to propagating modes. There can be population and polarization damping in the three-level system, which have the rates $\gamma_{\|}$and $\gamma_{\perp}$, respectively. In the theoretical description, we assume zero-temperature reservoirs.

Obviously, without the third excited state $|f\rangle$ (formally, we can set $g_{2}=0$ ), the model describes the damped-driven JC model. The vacuum Rabi splitting $2 g_{1}$ exceeds by $\sim 2$ orders of magnitude the linewidth of the dressed state resonances, $g_{1} \gg \kappa, \gamma_{\perp}, \gamma_{\|}$. Even the multiphoton transitions to higher-lying dressed states are far out of resonance when the system is driven at the bare resonator frequency, $\Delta_{1}=\Delta_{c}=0$. This setting favors the photon-blockade dim state up to large drive strengths.

Assume now that there is a very weak coupling of the state $|e\rangle$ to $|f\rangle$; that is, $0 \neq g_{2} \ll g_{1}$. Because of this latter relation, the JC spectrum associated with the two-level system $|g\rangle$ and $|e\rangle$ is only slightly perturbed and the corresponding dressed states $| \pm, n\rangle$ can be easily identified in the full spectrum, as shown in Fig. 2(a). The ground state is the $|g, 0\rangle$ state and the first excited state manifold is $| \pm, 1\rangle$ exhibiting the vacuum Rabi splitting $2 g_{1}$. Starting from the second excited manifold, there is a third level in each manifold that weakly couples to the JC dressed states. In these levels, because of the small coupling $g_{2} \ll g_{1}$, the dominant component is the atomic excitation $|f\rangle$. Therefore, the level spacing between these third levels $|f, n\rangle$ and the adjacent ones $|f, n \pm 1\rangle$ is uniform and resonant with the photon frequency $\omega$. This equidistant ladder in the spectrum has dramatic consequences and can eliminate the photon-blockade effect.

When the cavity is resonantly driven, the off-resonant states $| \pm, 2\rangle$ are weakly populated by two-photon transitions. A small fraction of this anyway small population 


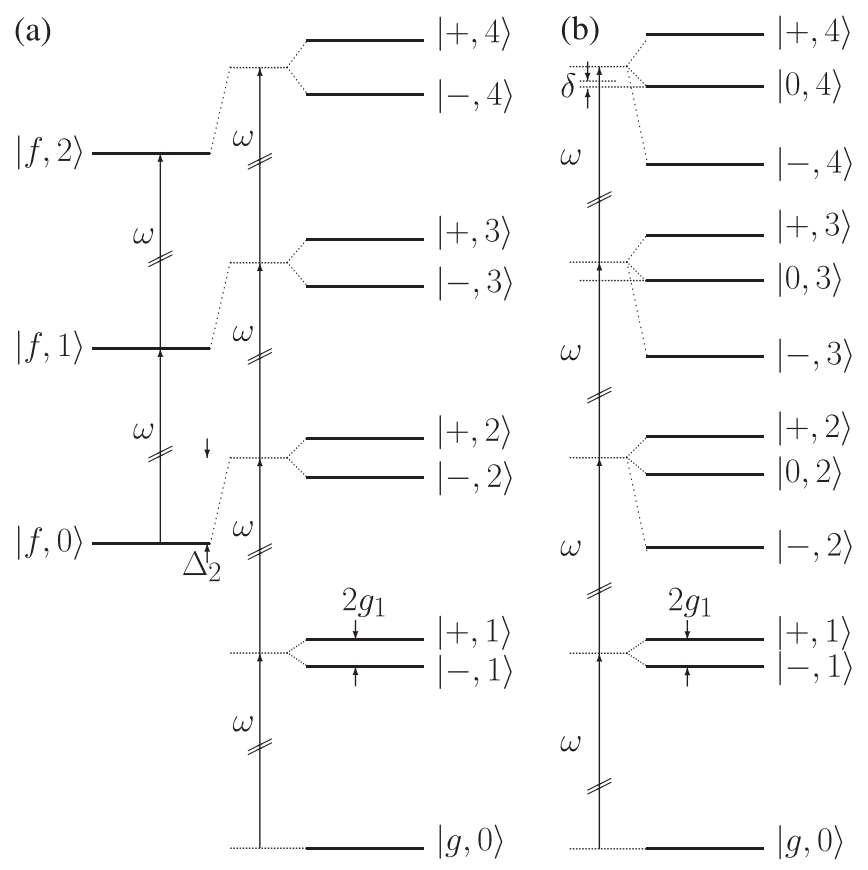

FIG. 2. (a) The spectrum of the coupled atom-cavity system if the third atomic state $|f\rangle$ is only weakly coupled to the middle one $|e\rangle$; that is, $g_{2} \ll g_{1}$. In (a), the $| \pm, n\rangle$ denote the usual twolevel atom JC model dressed states which combine the states $|g, n\rangle$ and $|e, n-1\rangle$. (b) The spectrum of the coupled atom-cavity system in the experiment is $\Delta_{1}=0, \Delta_{2} \simeq E_{C} / \hbar$, and $g_{2} \simeq \sqrt{2} g_{1}$. In (b), the multiplets $|\{-, 0,+\}, n\rangle$ correspond to the eigenstates of Eq. (1) in the manifold of a number of $n$ excitations. $\delta$ represents the mismatch of $\omega$ with respect to the transition frequency between the states $|0,3\rangle$ and $|0,4\rangle$, which is still an order of magnitude larger than the linewidth for these intermediate photon numbers.

leaks into this harmonic part of the spectrum, which is then resonantly driven within the manifold $|f, n\rangle$. This component of the wave function is thus coherently displaced in the photon mode on the time scale of $\kappa^{-1}$. The displacement is not interrupted by atomic decay since the polarization damping keeps the atomic state $|f\rangle$ intact, and the population decay from $|f\rangle$ to $|e\rangle$ is negligible on the excitation time scale, $\gamma_{\|}^{-1} \gg \kappa^{-1}$. The displacement is counteracted by cavity loss and leads to a steady state, which is a coherent state with an amplitude $2 \eta / \kappa$ and phase locked to the phase of the driving field. None of the loss processes lead out of this coherent excited state: (i) the cavity loss, together with the driving strength, sets the amplitude and does not break coherence; (ii) once the photon number is large enough, the change of the atomic state does not matter because the Jaynes-Cummings spectrum approaches a harmonic one also in the manifolds of states $|+, n\rangle$ and $|-, n\rangle$, and the phase is locked again to the phase of the drive $\eta$.

Just like in optical pumping, the total state of the system is gradually pumped into this trapping state, a coherent state of the resonator mode combined with a full mixture of the atomic states. The bottleneck formed by the initial two-photon transition and the weak $| \pm, 2\rangle \rightarrow|f, 0\rangle$ coupling increases only the time the system is pumped into this trapping state. The three-level atom in a cavity with the third level weakly coupled to the other two leads, thus, to a steady-state bright transmission identical to that of a resonantly driven empty cavity.

When the coupling $g_{2}$ is increased to $g_{1} \lesssim g_{2}$, the atomic state $|f\rangle$ mixes significantly with the other states $|g\rangle,|e\rangle$. The two atomic transitions coupled to the mode form a spectrum that can be sufficiently anharmonic in the lowexcitation part, as shown in Fig. 2(b), so that the system is blocked into the dim state up to high driving strengths at resonant driving $\Delta=0$,

Figure 3(a) presents a histogram of the calculated transmitted field intensity as a function of $g_{2}$ in the range between the two limiting cases, $g_{2}=0$ and $g_{1} \lesssim g_{2}$. As expected, small $g_{2}$ gives rise to a full transmission of the resonant driving power, and the photon number fluctuates around the mean $\left\langle a^{\dagger} a\right\rangle \approx(2 \eta / \kappa)^{2}=700$. Larger fluctuations and the residual population in the low-photon states, close to the origin $g_{2} \approx 0$ of the plot, are merely a finite simulation time effect. On the other hand, it is also confirmed that the three-level atom with $g_{2}>g_{1}$ switches off the transmission; i.e., it recovers the photon blockade for this drive strength. The histogram is prepared from the ensemble of intensity values recorded at many randomly chosen instants while the system is in steady state. The numerical simulations are performed using the $\mathrm{C}++\mathrm{QED}$ framework [41,42], which solves the master equation [Eq. (2)] by unraveling it into a set of Monte Carlo wave-function trajectories. The photon mode basis is truncated at the Fock state $n=3600$ and it takes several weeks to generate a histogram at a single value of $g_{2} / g_{1}$ by sampling a 0.5 -ms-long trajectory at every $\mu \mathrm{s}$.

The unexpected feature of the histogram in Fig. 3(a) is the transition domain manifested by a bimodal distribution of intensities. Experimentally, we are exactly in the center of this transition regime with $g_{2} / g_{1} \simeq \sqrt{2}$. Figure 3(b) presents measured vacuum Rabi spectra for different input probe powers varied over 4 orders of magnitude where all 3 transmons are in resonance with the cavity mode. Here, $P_{\text {in }}$ refers to the input power at the cavity input and $P_{\text {out }}$ refers to the digitizer input at room temperature. At low input powers corresponding to much less than a single intracavity photon on average, we observe the well-known splitting of the coupled multiqubit single-photon state [43]. The lack of additional resonances validates that the system is initialized in its quantum ground state rather than a thermal state [44]. At intermediate powers we observe a rich structure in the transmission spectrum, which is determined by multiqubit multiphoton transitions. The power broadening of the multiphoton resonances can be observed. The situation is even more complicated due to the additional transmon levels which lead to an asymmetry of the observed spectra $[5,6]$. More importantly, however, the frequency region 
(a)

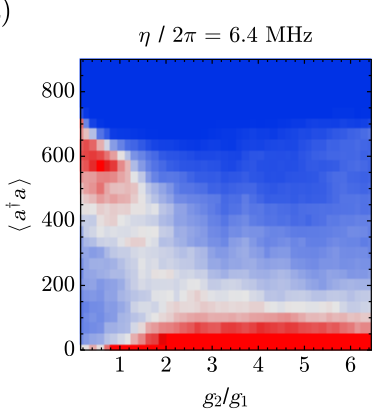

(c) $\quad \eta / 2 \pi(\mathrm{MHz})$

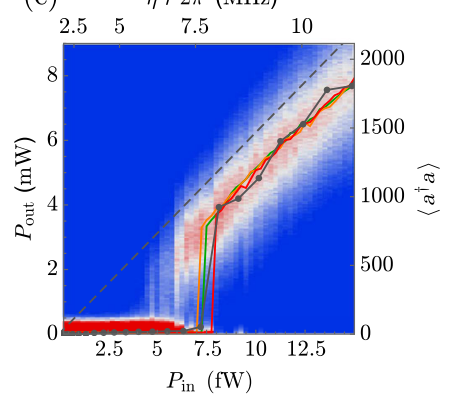

(b)

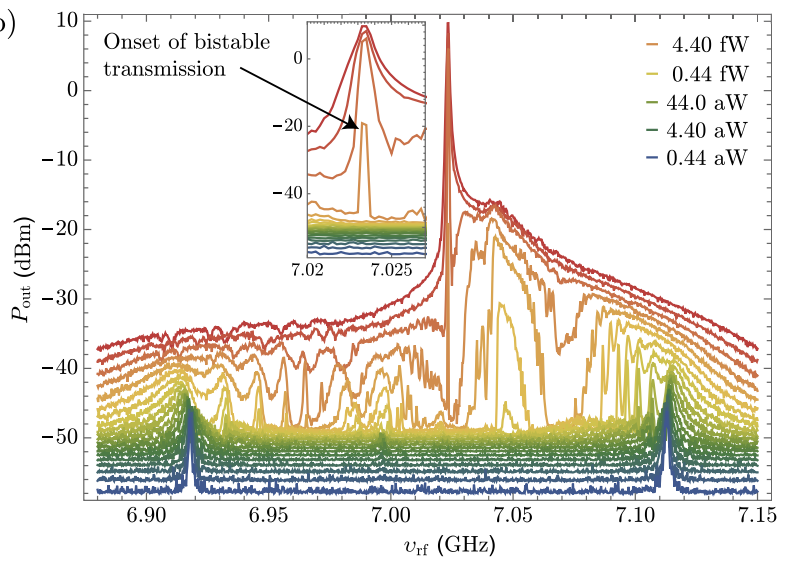

FIG. 3. (a) Simulated histogram of the output intensity as a function of the coupling constant $g_{2}$ between the two excited atomic states $|e\rangle$ and $|f\rangle$ for $\eta /(2 \pi)=6.4 \mathrm{MHz}$, with red representing high probability and blue indicating zero probablility. (b) Measured vacuum Rabi spectra for input powers from $P_{\text {in }}=0.44 \times 10^{-18} \mathrm{~W}$ (blue) to $25 \times 10^{-15} \mathrm{~W}$ (red) with all three atoms in resonance with the cavity. $P_{\text {in }}$ and $P_{\text {out }}$ refer to the cavity input power and digitizer input power, respectively. For better visibility the shown spectra are offset by $1.6 \mathrm{nW}$ from each other. The sharp transmission peak shown in the inset appears stochastically. In this particular measurement (orange line at $4.4 \mathrm{fW}$ input power), we observe only two frequency points with small but finite switching probability and we sample over multiple switching events resulting in a certain mean detected power. At lower drive power no transmission is observed (no switching). At higher drive powers the transmission peak approaches the cavity linewidth and scales linearly with input power (no switching again). (c) Measured histogram of the detected power as a function of the cavity input power for a single transmon (density plot). The most likely photon numbers (line plots) are extracted from this measurement (red) and two similar measurements taken with 2 (orange) and 3 qubits (green) in resonance with the cavity mode. Simulation results for the single qubit case are shown with connected black symbols for comparison. The dashed line is for reference and represents the response of the empty cavity with $\left\langle a^{\dagger} a\right\rangle=(2 \eta / \kappa)^{2}$.

around the bare cavity frequency remains dim over a large range of input powers. At a certain power, corresponding to about $n \sim 500$ intracavity photons, we observe a sudden narrow transmission peak at the bare cavity frequency. This transmission peak is observed to be switching stochastically for a certain range of constant

input powers. At much higher input powers, the spectrum resembles that of an empty cavity without atoms. This transition to the classical regime has been studied in the dispersive limit with coherent light where $n \sim 10^{5}$ [30], as well as in the resonant limit with broadband thermal radiation [45].

In order to resolve the dynamics of the process leading to the sharp transmission peak, we record the cavity transmission at the bare cavity frequency for 60 different input powers. In each case, we measure a single-shot real-time record of both field quadratures and repeat these measurements for one, two, and three transmons in resonance with the cavity mode. Experimentally, the transmitted tone is amplified with a commercial HEMT amplifier, downconverted with an IQ mixer and digitized with a time resolution of $40 \mathrm{~ns}$ for a total time of $1.6 \mathrm{~ms}$. This corresponds to a filter bandwidth of $25 \mathrm{MHz}$, much smaller than the vacuum Rabi splitting and small enough to filter out most low-lying and comparably low-power multiphoton transitions. The density plot shown in Fig. 3(c) shows the likelihood to detect a particular output power as a function of a large range of input powers, carefully mapping out the transition from the dim to the bright multiphoton phase of the system.

The measured histogram clearly shows an input power region between 4.5 and $7.5 \mathrm{fW}$ with two distinct solutions. For smaller powers, we observe no transmission (photon blockade), and for higher powers, the cavity transmission scales linearly with the input power (empty cavity solution). The red line plot in Fig. 3(c) indicates the most likely output power for this measurement and agrees very well with the simulated results shown in black. The simulation results are based on independently measured sample parameters, see also Ref. [39], and provide a calibration for the drive strength $\eta$ shown on the top horizontal axis of Fig. 3(c) and the mean intracavity photon number $\left\langle a^{\dagger} a\right\rangle$ shown on the right-hand vertical axis. The total attenuation of $86 \mathrm{~dB}$ agrees with our expectations based on independent measurements and is sufficient to protect the cavity from room-temperature thermal radiation. The orange and green lines in Fig. 3(c) show the results of the identical measurements with two and three atoms in resonance with the cavity mode. The characteristic switching power is slightly shifted, but no other differences are apparent between the single and multiatom case.

A full characterization of the steady state of the resonator field can be given by the Husimi $Q$ quasiprobability distribution; see Fig. 4. For small drive strength the system is in the photon-blockade regime and the $Q$ function is that of the vacuum state. Because of the added noise of the amplifier chain, we expect that a convolution of the $Q$ function vacuum state with a thermal state and a Gaussian fit to the measured distribution [see Fig. 4(a)] yields an added noise photon number of 28.1 as referenced to the cavity output. This corresponds to a total system noise 
(a)

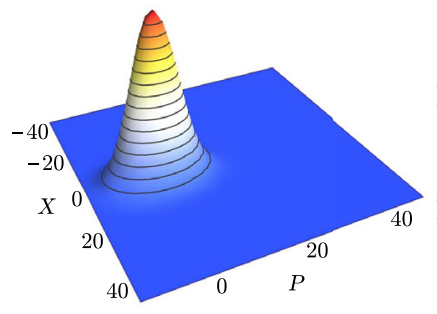

(c)

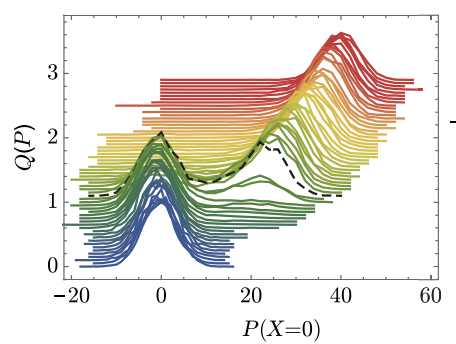

(e)

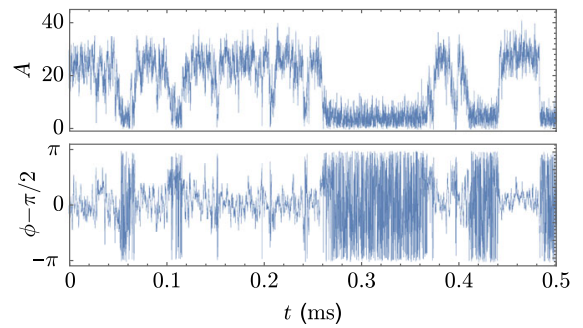

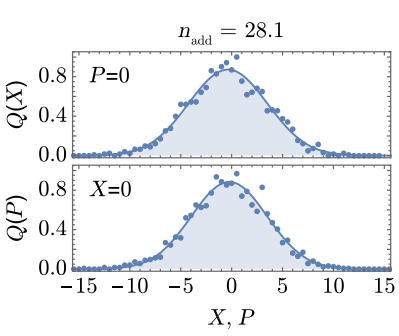

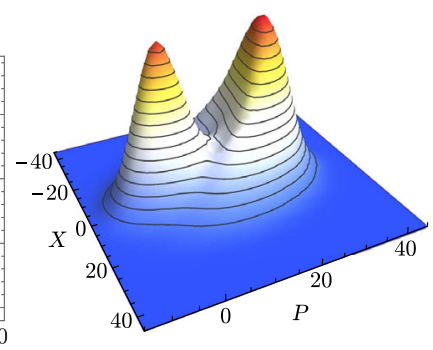

(b)
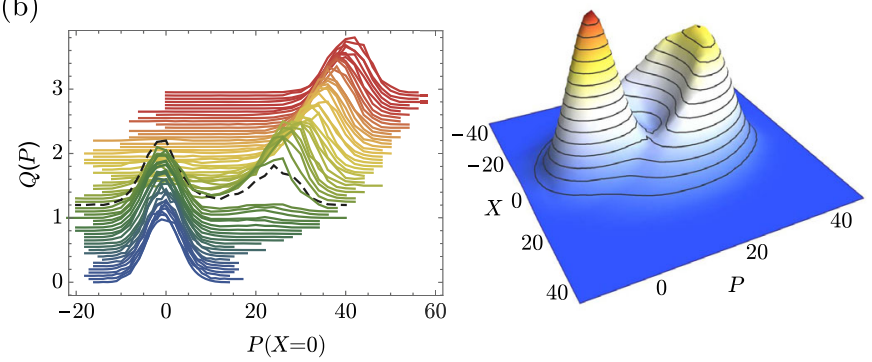

(d)
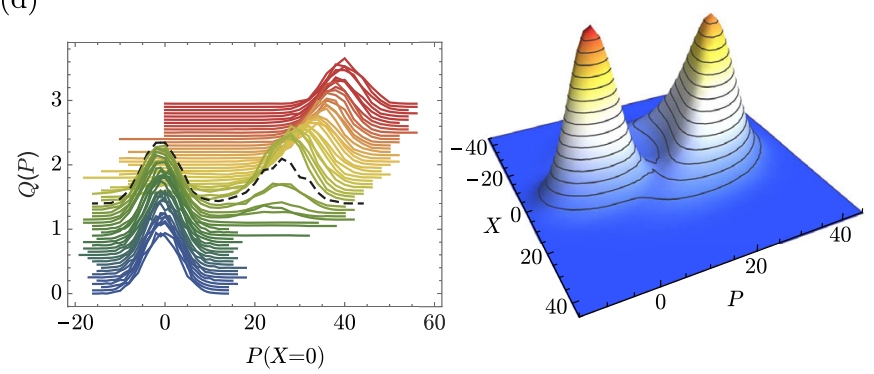

(g) (f)

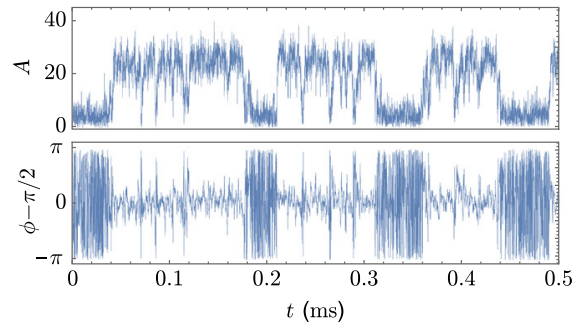

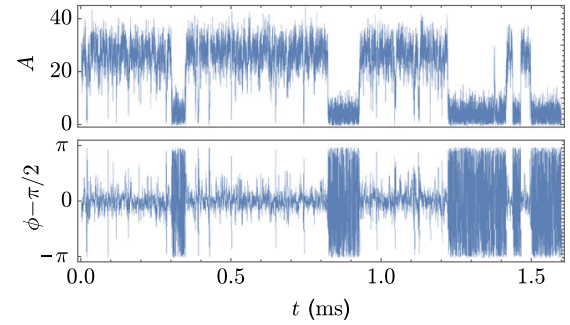

FIG. 4. (a) Measured $Q$ function of the vacuum field for very low drive power $\eta /(2 \pi)=1.4 \mathrm{MHz}$ in the photon-blockade regime (left) and Gaussian fit along the two field quadratures $X$ and $P$. The fit reveals a thermal state due to the added amplifier noise with $n_{\text {add }}=28.1$. (b) The waterfall plot shows line cuts of the measured $Q$ functions, where $X=0$ as the drive strength is increased from $\eta /(2 \pi)=1.4$ to $11 \mathrm{MHz}$ for a single transmon in resonance with the cavity (left). A vertical offset of 0.05 between the line plots is used for better visibility. The complete $Q$ function for the critical drive strength $\eta /(2 \pi)=7.1 \mathrm{MHz}$, which is indicated by the dashed black line on the left. (c) The same measurement for transmons 1 and 2 in resonance and the critical drive strength $\eta /(2 \pi)=6.8 \mathrm{MHz}$. (d) The same for transmons 1, 2, and 3 in resonance and the critical drive strength $\eta /(2 \pi)=7.7 \mathrm{MHz}$. (e)-(g) The real-time single-shot record of the transmitted output field amplitude and phase for one (e), two (f), and three (g) atoms in resonance with the cavity.

temperature of $9.5 \mathrm{~K}$, in line with our expectations due to losses in the output line and the used amplifiers' noise figures.

Figures 4(b), 4(c), and 4(d) show the experimental results for one, two, and three atoms in resonance, respectively. The waterfall plots explicitly show the photon-blockade breaking transition indicated by the sudden change of the $Q$-function peak position as the drive power is increased from low (blue) to high drive strength (red). The right-hand plots depict the entire $Q$ function close to the critical point, i.e., for the data sets marked by dashed black lines on their left. In all three cases, the $Q$ function shows a clear bimodal structure with two solutions. Because of the large intracavity photon number in the bimodal switching regime of $\sim 500-700$, we can clearly resolve the double-peaked $Q$ function demonstrating the mixture of the vacuum state and the highly excited state with a well-defined amplitude and phase. From the better-resolved $Q$ function in the three-atom case compared to the single- and two-atom cases, it appears that the phase stability is improved for larger systems.

The observed (bi)stability can be maintained only for a finite time and there is a substantial difference with respect to the classical bistability. Within the quantum domain, such as in the microscopic medium composed by a single atom, the branches are not stable on macroscopic time scales. Quantum fluctuations in a finite system lead to diffusion such that the system can leave the robust semiclassical attractors in a finite duration of time. This is shown in Figs. 4(e)-4(g), which present the time evolution of the system in steady state. The system flips between two quasistationary solutions corresponding to the two components of the bimodal $Q$ function: the vacuum with an undefined phase and an excited coherent state. The wellresolved plateaus reveal that the dwell time in each of these components is longer then the microscopic time scales 
characteristic for equilibration. The typical time scale is expected to depend on the system size and would increase to infinity for a macroscopic system [46]. Experimentally, we find a much longer characteristic switching time of $\sim 0.5 \mathrm{~ms}$ when all three transmons are tuned in resonance with the microwave resonator, see Fig. 4(g), compared to the time dynamics with only one [Fig. 4(e)] or two atoms [Fig. 4(f)], where the typical switching time is on the order of $\sim 0.1 \mathrm{~ms}$. In all cases, the typical time scales exceed by far the cavity and qubit coherence times of 0.1-0.4 $\mu$ s [39].

In summary, we study the nonequilibrium first-order phase transition that takes place when a strongly driven quantum systems breaks through the nonresonant, reflective phase into a transmissive excited one. We show both theoretically and experimentally that the presence of an additional near-resonant atomic level dramatically changes the system dynamics and allows us to access the excited phase. In a given range of driving strengths, the transition gives rise to a mixture of the two phases, and the quantum system is switching between the two corresponding states. The increase of the dwell time as the size of the system is enlarged, from one to three atoms in our experiment, is observed, and points to the stabilization of the corresponding phases in the classical limit.

Our results represent an important first step towards exploring quantum criticality in the many-photon-highdrive-power regime. A detailed understanding of quantum phase transitions in circuit QED [47,48] could play an important role for the controlled simulation and stabilization of peculiar phases of finite-size open quantum systems. In the future, one could study entanglement [49] and the details of the time dynamics using autocorrelation measurements [11]. With better qubit coherence times and quantum-limited amplifiers, phase multistability [50] and squeezing [51] may be realizable. Another potential direction is the application as an ultralow energy classical set reset flip-flop memory for ultralow power signal processing in photonics [52] or classical front-end processing for quantum computers [53].

We thank A. Blais for comments on the manuscript. This work was supported by ETH Zurich, IST Austria, the Hungarian Academy of Sciences (Lendület Program, LP2011-016), and the National Research, Development and Innovation Office (K115624). A. V. acknowledges support from the János Bolyai Research Scholarship of the Hungarian Academy of Sciences. We acknowledge NIIF for awarding us access to the computational resource based in Hungary at Debrecen.

[1] E. T. Jaynes and F. W. Cummings, Comparison of Quantum and Semiclassical Radiation Theories with Application to the Beam Maser, Proc. IEEE 51, 89 (1963).
[2] S. Haroche and J.-M. Raimond, Exploring the Quantum: Atoms, Cavities, and Photons (Oxford University Press, New York, 2006).

[3] A. Wallraff, D. I. Schuster, A. Blais, L. Frunzio, R.-S. Huang, J. Majer, S. Kumar, S. M. Girvin, and R. J. Schoelkopf, Strong Coupling of a Single Photon to a Superconducting Qubit Using Circuit Quantum Electrodynamics, Nature (London) 431, 162 (2004).

[4] I. Schuster, A. Kubanek, A. Fuhrmanek, T. Puppe, P. W. H. Pinkse, K. Murr, and G. Rempe, Nonlinear Spectroscopy of Photons Bound to One Atom, Nat. Phys. 4, 382 (2008).

[5] J. M. Fink, M. Göppl, M. Baur, R. Bianchetti, P. J. Leek, A. Blais, and A. Wallraff, Climbing the Jaynes-Cummings Ladder and Observing Its Nonlinearity in a Cavity QED System, Nature (London) 454, 315 (2008).

[6] L.S. Bishop, J. M. Chow, J. Koch, A. A. Houck, M. H. Devoret, E. Thuneberg, S. M. Girvin, and R. J. Schoelkopf, Nonlinear Response of the Vacuum Rabi Resonance, Nat. Phys. 5, 105 (2009).

[7] S. S. Shamailov, A. S. Parkins, M. J. Collett, and H. J. Carmichael, Multi-Photon Blockade and Dressing of the Dressed States, Opt. Commun. 283, 766 (2010).

[8] A. Imamoglu, H. Schmidt, G. Woods, and M. Deutsch, Strongly Interacting Photons in a Nonlinear Cavity, Phys. Rev. Lett. 79, 1467 (1997).

[9] K. M. Birnbaum, A. Boca, R. Miller, A. D. Boozer, T. E. Northup, and H. Jeff Kimble, Photon Blockade in an Optical Cavity with One Trapped Atom, Nature (London) 436, 87 (2005).

[10] A. Faraon, I. Fushman, D. Englund, N. Stoltz, P. Petroff, and J. Vuckovic, Coherent Generation of Non-Classical Light on a Chip via Photon-Induced Tunnelling and Blockade, Nat. Phys. 4, 859 (2008).

[11] C. Lang, D. Bozyigit, C. Eichler, L. Steffen, J. M. Fink, A. A. Abdumalikov, M. Baur, S. Filipp, M. P. da Silva, A. Blais, and A. Wallraff, Observation of Resonant Photon Blockade at Microwave Frequencies Using Correlation Function Measurements, Phys. Rev. Lett. 106, 243601 (2011).

[12] A. Kubanek, A. Ourjoumtsev, I. Schuster, M. Koch, P. W. H. Pinkse, K. Murr, and G. Rempe, Two-Photon Gateway in One-Atom Cavity Quantum Electrodynamics, Phys. Rev. Lett. 101, 203602 (2008).

[13] A. Dombi, A. Vukics, and P. Domokos, Bistability Effect in the Extreme Strong Coupling Regime of the JaynesCummings Model, Eur. Phys. J. D 69, 60 (2015).

[14] H. J. Carmichael, Breakdown of Photon Blockade: A Dissipative Quantum Phase Transition in Zero Dimensions, Phys. Rev. X 5, 031028 (2015).

[15] D. Nagy, G. Szirmai, and P. Domokos, Self-Organization of a Bose-Einstein Condensate in an Optical Cavity, Eur. Phys. J. D 48, 127 (2008).

[16] K. Baumann, C. Guerlin, F. Brennecke, and T. Esslinger, Dicke Quantum Phase Transition with a Superfluid Gas in an Optical Cavity, Nature (London) 464, 1301 (2010).

[17] D. Dagnino, N. Barberan, M. Lewenstein, and J. Dalibard, Vortex Nucleation as a Case Study of Symmetry Breaking in Quantum Systems, Nat. Phys. 5, 431 (2009).

[18] A. Pályi, P. R. Struck, M. Rudner, K. Flensberg, and G. Burkard, Spin-Orbit-Induced Strong Coupling of a Single 
Spin to a Nanomechanical Resonator, Phys. Rev. Lett. 108, 206811 (2012).

[19] A. Trenkwalder, G. Spagnolli, G. Semeghini, S. Coop, M. Landini, P. Castilho, L. Pezze, G. Modugno, M. Inguscio, A. Smerzi, and M. Fattori, Quantum Phase Transitions with Parity-Symmetry Breaking and Hysteresis, Nat. Phys. 12, 826 (2016).

[20] S. R. K. Rodriguez, W. Casteels, F. Storme, I. Sagnes, L. Le Gratiet, E. Galopin, A. Lemaitre, A. Amo, C. Ciuti, and J. Bloch, Dynamic Optical Hysteresis in the Quantum Regime, arXiv:1608.00260.

[21] M. Fitzpatrick, N. M. Sundaresan, A. C. Y. Li, J. Koch, and A. A. Houck, Observation of a Dissipative Phase Transition in a One-Dimensional Circuit QED Lattice, arXiv:1607.06895.

[22] M.-J. Hwang, R. Puebla, and M. B. Plenio, Quantum Phase Transition and Universal Dynamics in the Rabi Model, Phys. Rev. Lett. 115, 180404 (2015).

[23] M.-J. Hwang and M. B. Plenio, Quantum Phase Transition in the Finite Jaynes-Cummings Lattice Systems, Phys. Rev. Lett. 117, 123602 (2016).

[24] A. Dombi, A. Vukics, and P. Domokos, Optical Bistability in Strong-Coupling Cavity QED with a Few Atoms, J. Phys. B 46, 224010 (2013).

[25] A. T. Rosenberger, L. A. Orozco, H. J. Kimble, and P. D. Drummond, Absorptive Optical Bistability in Two-State Atoms, Phys. Rev. A 43, 6284 (1991).

[26] G. Rempe, R. J. Thompson, R. J. Brecha, W. D. Lee, and H. J. Kimble, Optical Bistability and Photon Statistics in Cavity Quantum Electrodynamics, Phys. Rev. Lett. 67, 1727 (1991).

[27] C. M. Savage and H. J. Carmichael, Single Atom Optical Bistability, IEEE J. Quantum Electron. 24, 1495 (1988).

[28] J. Kerckhoff, M. A. Armen, and H. Mabuchi, Remnants of Semiclassical Bistability in the Few-Photon Regime of Cavity QED, Opt. Express 19, 24468 (2011).

[29] E. Ginossar, L. S. Bishop, D. I. Schuster, and S. M. Girvin, Protocol for High-Fidelity Readout in the Photon-Blockade Regime of Circuit QED, Phys. Rev. A 82, 022335 (2010).

[30] M. D. Reed, L. DiCarlo, B. R. Johnson, L. Sun, D. I. Schuster, L. Frunzio, and R. J. Schoelkopf, High-Fidelity Readout in Circuit Quantum Electrodynamics Using the Jaynes-Cummings Nonlinearity, Phys. Rev. Lett. 105, 173601 (2010).

[31] L. S. Bishop, E. Ginossar, and S. M. Girvin, Response of the Strongly Driven Jaynes-Cummings Oscillator, Phys. Rev. Lett. 105, 100505 (2010).

[32] M. Boissonneault, J. M. Gambetta, and A. Blais, Improved Superconducting Qubit Readout by QubitInduced Nonlinearities, Phys. Rev. Lett. 105, 100504 (2010).

[33] R. Vijay, M. H. Devoret, and I. Siddiqi, Invited Review Article: The Josephson Bifurcation Amplifier, Rev. Sci. Instrum. 80, 111101 (2009).

[34] K. W. Murch, E. Ginossar, S. J. Weber, R. Vijay, S. M. Girvin, and I. Siddiqi, Quantum State Sensitivity of an Autoresonant Superconducting Circuit, Phys. Rev. B 86, 220503 (2012).
[35] P. Alsing and H.J. Carmichael, Spontaneous DressedState Polarization of a Coupled Atom and Cavity Mode, Quantum Opt. 3, 13 (1991).

[36] M. A. Armen, A. E. Miller, and H. Mabuchi, Spontaneous Dressed-State Polarization in the Strong Driving Regime of Cavity QED, Phys. Rev. Lett. 103, 173601 (2009).

[37] J. Raftery, D. Sadri, S. Schmidt, H. E. Türeci, and A. A. Houck, Observation of a Dissipation-Induced Classical to Quantum Transition, Phys. Rev. X 4, 031043 (2014).

[38] J. M. Fink, R. Bianchetti, M. Baur, M. Göppl, L. Steffen, S. Filipp, P. J. Leek, A. Blais, and A. Wallraff, Dressed Collective Qubit States and the Tavis-Cummings Model in Circuit QED, Phys. Rev. Lett. 103, 083601 (2009).

[39] J. A. Mlynek, A. A. Abdumalikov, J. M. Fink, L. Steffen, M. Baur, C. Lang, A. F. van Loo, and A. Wallraff, Demonstrating W-Type Entanglement of Dicke States in Resonant Cavity Quantum Electrodynamics, Phys. Rev. A 86, 053838 (2012).

[40] J. Koch, T. M. Yu, J. Gambetta, A. A. Houck, D. I. Schuster, J. Majer, A. Blais, M. H. Devoret, S. M. Girvin, and R. J. Schoelkopf, Charge-Insensitive Qubit Design Derived from the Cooper Pair Box, Phys. Rev. A 76, 042319 (2007).

[41] A. Vukics and H. Ritsch, C++QED: An Object-Oriented Framework for Wave-Function Simulations of Cavity QED Systems, Eur. Phys. J. D 44, 585 (2007).

[42] A. Vukics, $C++Q E D v 2$ : The Multi-Array Concept and Compile-Time Algorithms in the Definition of Composite Quantum Systems, Comput. Phys. Commun. 183, 1381 (2012).

[43] G. S. Agarwal, Vacuum-Field Rabi Splittings in Microwave Absorption by Rydberg Atoms in a Cavity, Phys. Rev. Lett. 53, 1732 (1984).

[44] J. M. Fink, M. Baur, R. Bianchetti, S. Filipp, M. Göppl, P. J. Leek, L. Steffen, A. Blais, and A. Wallraff, Thermal Excitation of Multi-Photon Dressed States in Circuit Quantum Electrodynamics, Phys. Scr. T137, 014013 (2009).

[45] J. M. Fink, L. Steffen, P. Studer, L. S. Bishop, M. Baur, R. Bianchetti, D. Bozyigit, C. Lang, S. Filipp, P. J. Leek, and A. Wallraff, Quantum-to-Classical Transition in Cavity Quantum Electrodynamics, Phys. Rev. Lett. 105, 163601 (2010).

[46] W. Casteels, R. Fazio, and C. Ciuti, Critical Scaling of the Liouvillian Gap for a Nonlinear Driven-Dissipative Resonator, arXiv:1608.00717 [Phys. Rev. A (to be published)].

[47] M. Feng, Y. P. Zhong, T. Liu, L. L. Yan, W. L. Yang, J. Twamley, and H. Wang, Exploring the Quantum Critical Behaviour in a Driven Tavis-Cummings Circuit, Nat. Commun. 6, 7111 (2015).

[48] L. Tian, Circuit QED and Sudden Phase Switching in a Superconducting Qubit Array, Phys. Rev. Lett. 105, 167001 (2010).

[49] R. Rogers, N. Cummings, L. Pedrotti, and P. Rice, AtomField Entanglement in Cavity QED: Nonlinearity and Saturation, arXiv:1512.00936.

[50] M. Delanty, S. Rebić, and J. Twamley, Superradiance and Phase Multistability in Circuit Quantum Electrodynamics, New J. Phys. 13, 053032 (2011). 
[51] V. Peano and M. Thorwart, Dynamical Bistability in the Driven Circuit QED, Europhys. Lett. 89, 17008 (2010).

[52] J. Kerckhoff, M. A. Armen, D.S. Pavlichin, and H. Mabuchi, The Dressed Atom as Binary Phase Modulator:
Towards Attojoule/Edge Optical Phase-Shift Keying, Opt. Express 19, 6478 (2011).

[53] C. K. Andersen and K. Mølmer, Circuit QED Flip-Flop Memory with All-Microwave Switching, Phys. Rev. Applied 3, 024002 (2015). 